\title{
Athletic Injuries to the Elbow, Forearm, Wrist and Hand
}

\author{
C.art. F. Badgify, m.d.* and .Iohn T. Hayfs, m.d., † Ann Arbor, Micbigan
}

\begin{abstract}
Lthough competitive sports produce more $A_{\text {major injuries to the lower extremity, a }}$ sufficient number of injuries to the upper extremity do occur to make a thorough review of their recognition and management worthwhile. Furthermore, since most of these injuries involve one of the major joints, such as the elbow or the wrist, inadequate treatment will often lead to permanent disability.

A number of factors are present which determine the type of injury sustained. Such factors are: the type of game (e.g., football, baseball, wrestling), experience and conditioning of the players, the type of forces applied to the extremity and the age of the player. Since the vast majority of college athletes are beyond the age of epiphyseal closure, it is obvious that epiphyseal injuries are rarely seen in the college group, whereas, they are common in the junior high school and high school group. Baseball produces frequent injuries to the fingers and thumb, while football and wres-
\end{abstract}
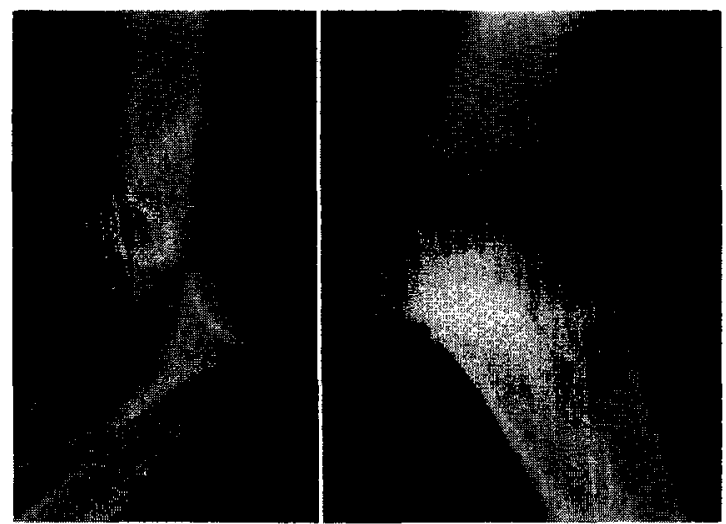

FIg. I. Simple posterior dislocation of the left elbow without fracture. Lateral view. tling produce more injuries to the elbow and wrist.

Management of athletic injuries on the Orthopaedic Surgery Service at The University of Michigan Hospital includes experience with injuries sustained in professional, intercollegiate, university intramural, high school, junior high school and "sand-lot" games. Players from every recognized collegiate or scholastic sport have been treated and the program outlined herein is based on this cumulative experience. These injuries will be presented according to their location.

\section{INJURIES OF THE ELBOW}

Dislocation of the elbow is one of the most common injuries to the upper extremity and has been encountered most often in injuries sustained in football. Other sports responsible for dislocations of the elbow have been wrestling, hockey, baseball and gymnastics. The diagnosis of dislocation of the elbow is usually not difficult. The mechanism is often a twisting or hyperextension injury. The player experiences immediate pain and loss of motion.

Examination will reveal lack of mobility of the elbow and deformity. Careful palpation of the bony prominences will disclose derangement of their normal relationships. Most dislocations of the elbow will fall into two groups. The first group includes the relatively simple posterior dislocation with minor ligamentous damage. The olecranon process is found thrust posteriorly along with the radial head. The radial head will be absent from its normal location just distal to the Iateral epicondyle of the humerus. There is little or no lateral or medial displacement. (Fig. r.) The radial and ulnar collateral ligaments are relatively intact and

* Professor of Surgery, The University of Michigan Medical Center, Ann Arbor, Michigan. $\dagger$ Assistant Professor of Surgery, The University of Michigan Medical Center, Ann Arbor, Michigan. 
Athletic Injuries to Elbow, Forearm, Wrist and Hand

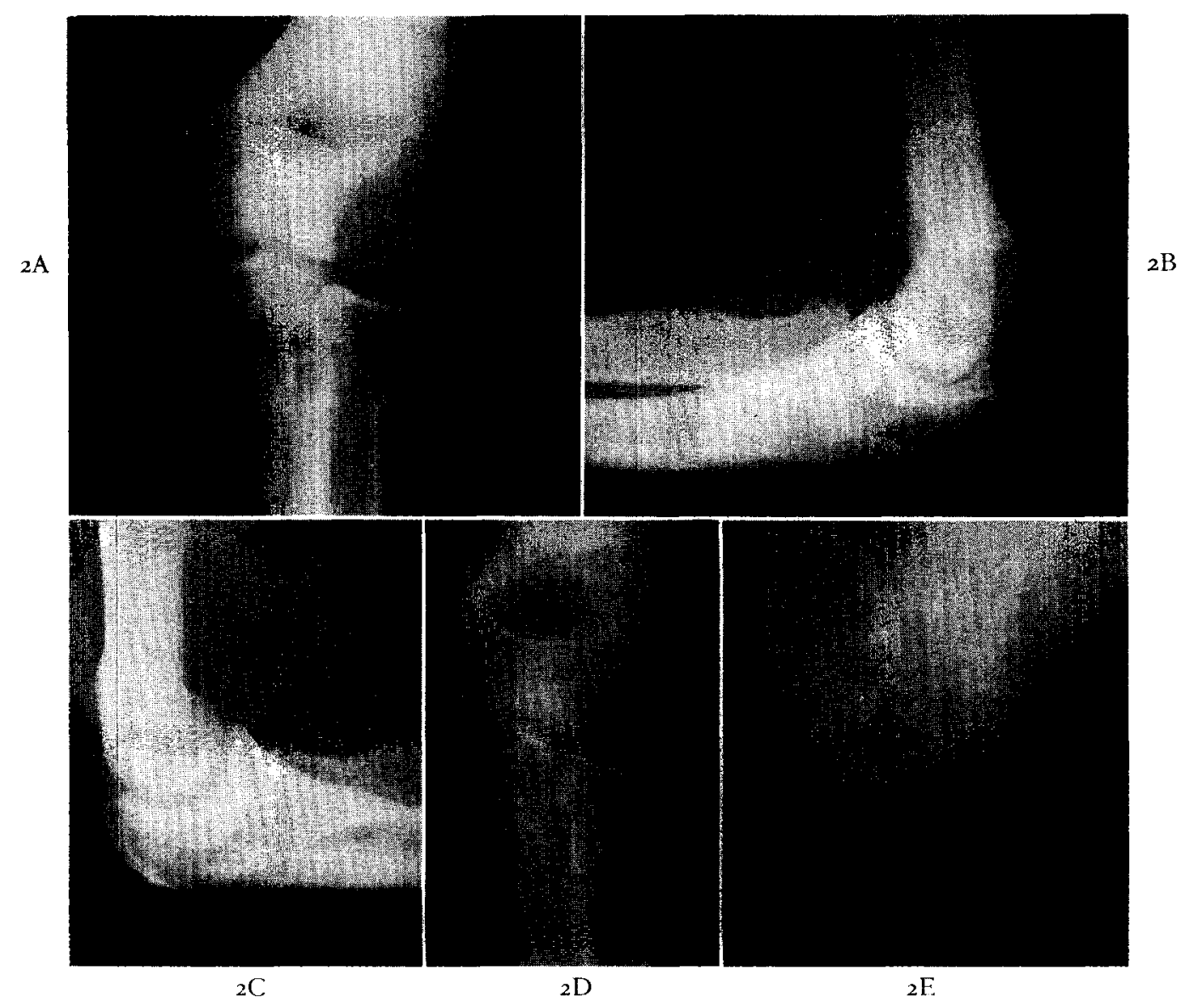

Fig. 2. A and B, football injury with posteromedial dislocation of the elbow. The dislocation was reduced on the playing field. Roentgenograms show the massive swelling secondary to hemorrhage. $\mathrm{C}, \mathrm{D}$ and $\mathrm{E}$, two months after injury. Calcification is present over the medial and lateral humeral epicondyles and in the olecranon fossa. The range of motion is normal except for a 20 degree loss of extension.

reduction is accompanied by a palpable and sometimes audible snap. Reduction may be accomplished with simple analgesia such as the intravenous administration of Demero ${ }^{(\circledast)}$ although general anesthesia may be necessary.

The finding of relatively intact collateral ligaments is confirmed by the relative stability of the elbow noted after reduction. In such a dislocation, splinting is carried out only as long as pain and swelling are present; active range of motion exercises are begun early, often during the first week after injury. Between exercise periods, a posterior splint may be worn with the elbow at a right angle. After three weeks, the splint may be discontinued.

Prior to epiphyseal closure, the medial humeral epicondyle is weakly attached to the humeral shaft and is frequently avulsed when the elbow is dislocated. All degrees of displacement of the medial humeral epicondylar epiphysis may occur. The most marked displacement occurs in posterolateral dislocation of the elbow in which the medial humeral epicondyle may be widely separated from its bed and drawn laterally into the elbow joint.

A second group of dislocations of the elbow are those with associated medial or lateral displacement. These injuries are more severe and are associated with complete rupture of the collateral ligaments of the elbow. Massive swelling and hemorrhage are invariably found. (Fig. 2.) Dislocations of this type with the attendant loss of collateral ligamentous continuity are quite unstable. Reduction is char- 


\section{Badgley and Hayes}

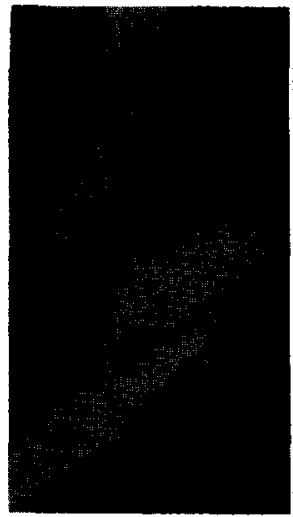

$3 \mathrm{~A}$

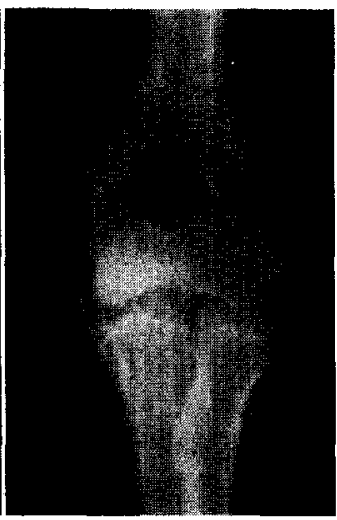

$3 \mathrm{~B}$

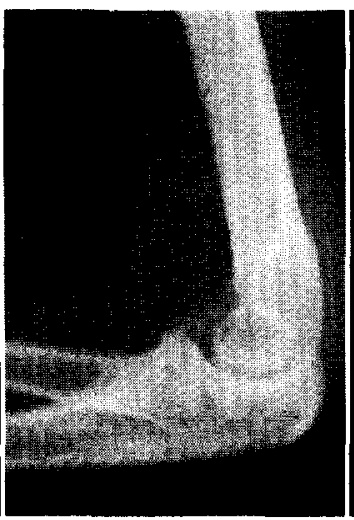

${ }_{3} \mathrm{C}$

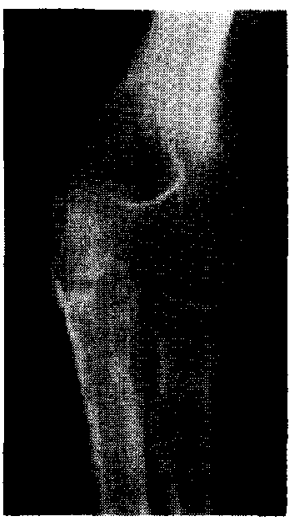

$3 \mathrm{D}$

FIG. 3. A, posterolateral dislocation of the elbow. B, after reduction. Small avulsion fractures of the medial and lateral humeral epicondyles are visible. C, three months after injury. A small area of myositis ossificans is present near the coronoid process of the ulna. D, three months after injury. The avulsion fragments are barely visible,

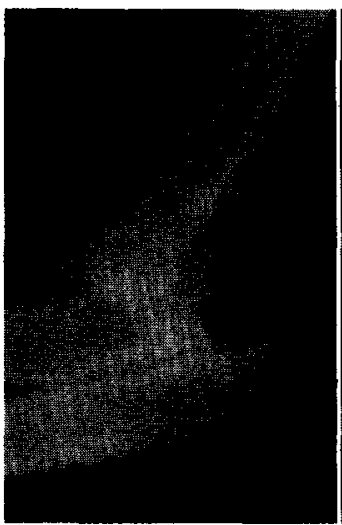

$4 \mathrm{~A}$

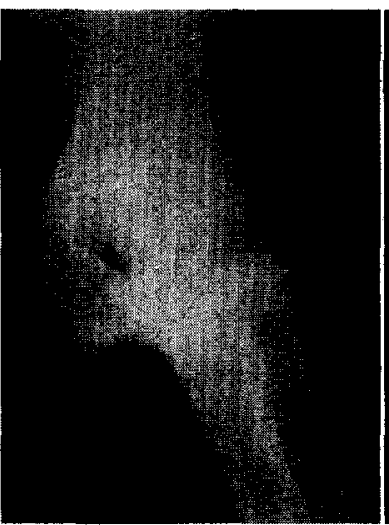

$4 \mathrm{~B}$

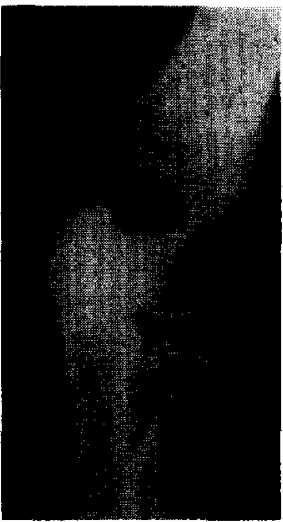

${ }_{4} \mathrm{C}$

Fig. 4. A and B, posterolateral dislocation of the elbow. C, after reduction. A comminuted fracture of the lateral humeral condyle is visible.

acterized by a tendency for the parts to move easily together and to redisplace with similar ease. Although this type of dislocation of the elbow must be splinted adequately to prevent redislocation and to allow healing of the torn collateral ligaments, early active motion is very important to restore range of motion. Usually, active motion is begun after three weeks of immobilization in a cast. Simple flexion, extension, pronation and supination movements can be begun under supervision. Between exercise periods, the elbow may be supported by a posterior splint. After the fourth week, splinting may be gradually discontinued.

Posterior dislocation of the elbow may be associated with a fracture involving the radial head, capitulum, or the coronoid process of the ulna. Medial or lateral dislocation may be associated with fracture of either the medial or Iateral epicondyle of the humerus. (Figs, 3 and 4.) Very small fragments may be ignored while larger ones may need to be excised. Fragments of these structures may be included in the joint with reduction and should be removed if such an inclusion should occur. (Fig. 5.)

Myositis ossificans is a common complication of dislocation of the elbow and is most often found in the region of the insertion of the brachialis muscle into the coronoid portion of the ulna. (Fig. ${ }_{3}$ C.) Myositis ossificans may 
Athletic Injuries to Elbow, Forearm, Wrist and Hand

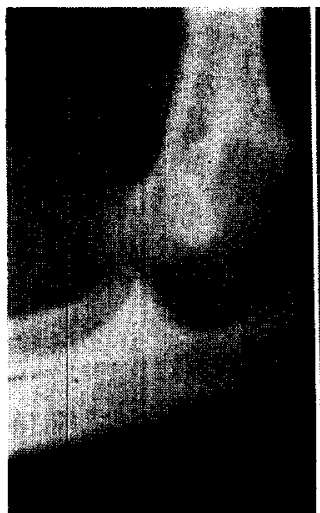

$5 \mathrm{~A}$

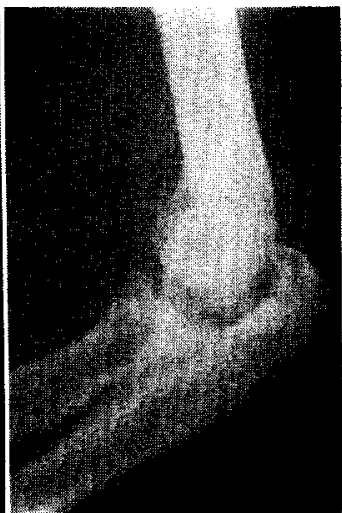

;B

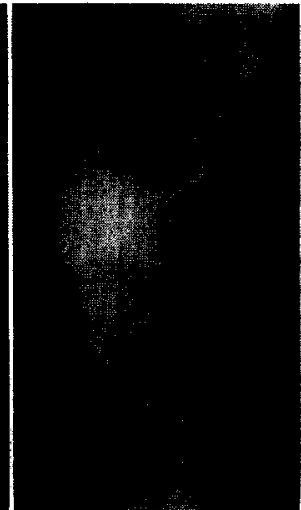

¡C

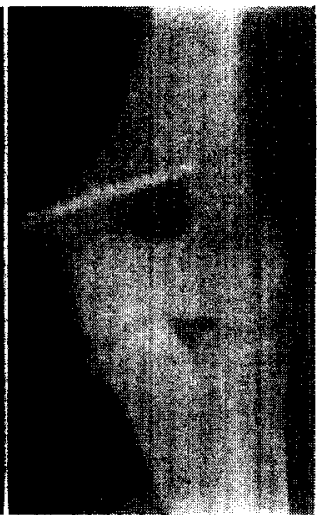

$; \mathrm{D}$

Fic. ;. A, fracture dislocation of the elbow in a fifteen year old boy. The medial humeral epicondylar epiphysis has been avulsed. B and C, following closed reduction. The medial humeral cpicondylar epiphysis is included in the elbow joint. D, after open reduction and internal frxation of the medial humeral epicondyle. The ulnar nerve was transplanted anteriorly.

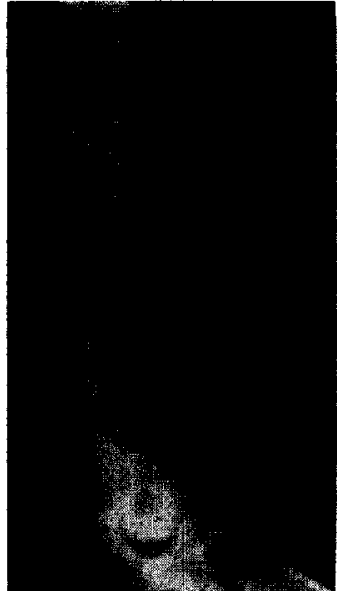

$6 \mathrm{~A}$

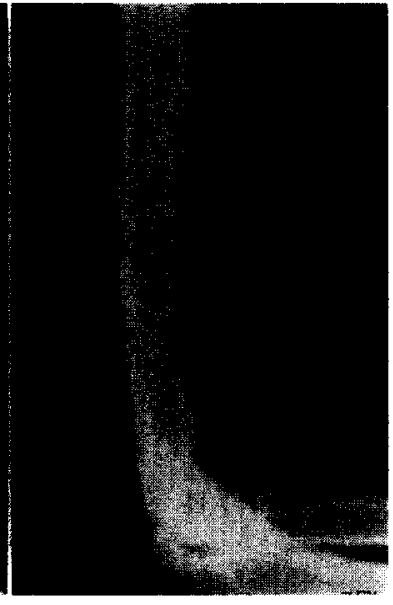

$6 \mathrm{~B}$
FIG. 6. A, myositis ossificans in the brachialis muscle. $B$, nine months later. The myositis ossificans has matured.

occur in other areas about the elbow, for example, in the region of the medial and lateral epicondyles. (Figs. 2C, 2D and 6.)

Myositis ossificans occurs in the region of the elbow more commonly than about any other joint and may be seen following injury to or operative intervention in the elbow. Murray [ $I]$ has cautioned against poorly timed operations on the elbow as an exciting factor in the production of calcification. He described the time sequence in the reaction of soft tissue about an injured joint. He believed that open operative procedures should be performed within the first few hours; otherwise, operation

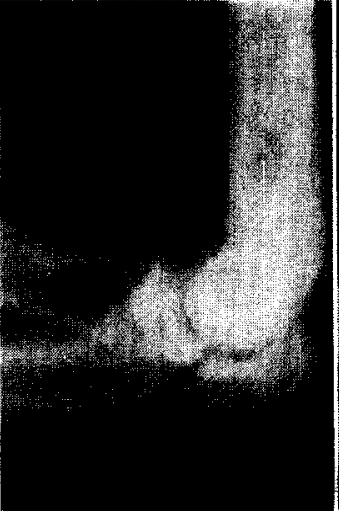

$7 \mathrm{~A}$

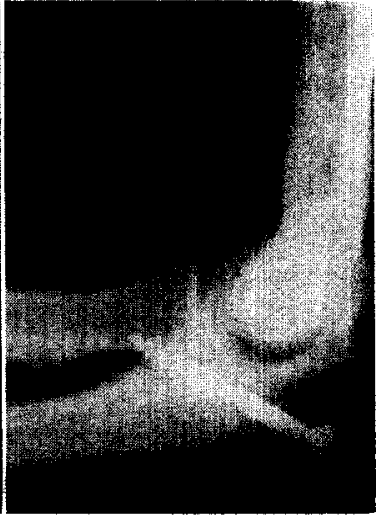

$7 \mathrm{~B}$
FIG. 7. A, fracture of the olecranon of the ulna involving the elbow joint. $B$, following open reduction and internal fixation of the olecranon.

should be postponed until all evidence of a pathologic condition of the soft parts has disappeared. The waiting period is usually two to three months or Ionger. Operation carried out after the reaction of the soft parts has reached its peak will increase the tendency to calcification and ossification.

Direct trauma to the elbow may produce a fracture of the olecranon process. The triceps muscle exerts a deforming force on this fracture with separation of the fragments. Open reduction with internal fixation is the preferred method of management of the displaced olecranon fracture. (Fig. 7.) Firm fixation of the fracture allows very early active motion of the elbow and avoids the necessity of immobilizing 


\section{Badgley and Hayes}

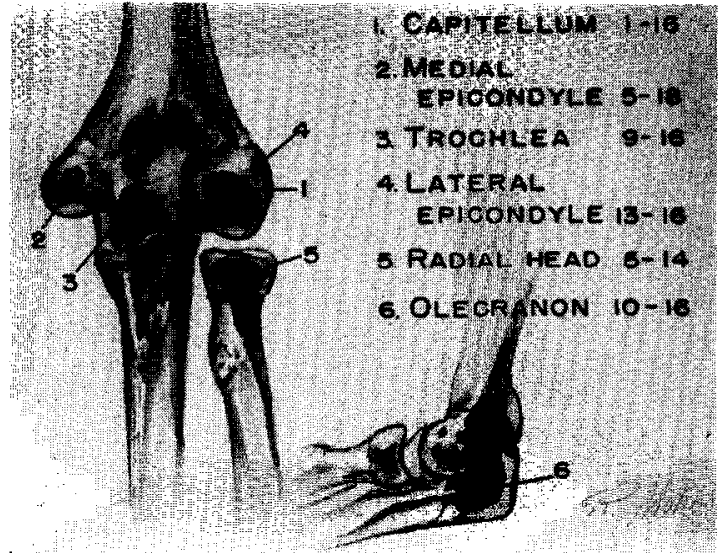

Fig. 8. Growth centers about the elbow. The approximate age of appearance of the centers and their fusion to the humcral shaft is given.

the elbow in extension. The head of the radius is frequently fractured when impact forces are transmitted from the hand and forearm to the elbow. Relatively undisplaced stellate fractures of the radial head are treated by splinting of the elbow at a right angle. Active exercise is instituted as soon as pain subsides. Comminuted and displaced fractures of the radial head arc trcatcd by excision.

With the introduction of organized competitive sports into the junior high school program, more and more epiphyseal injuries can be anticipated. Injuries of the elbow in junior high school football must be evaluated with respect to the development of the ossiffcation centers of the distal part of the humerus and the proximal part of the ulna and radius. The distal end of the humerus ossifies in four centers. The centers for the capitulum and the trochlea coalesce into a single mass of bone at ten to twelve years. However, the ossification centers for the lateral and medial epicondyles remain separated from the humeral shaft until just prior to the age of epiphyseal closure. (Fig. 8.) Fractures of the capitulum with lateral rotation and displacement of the capitulum are common below the age of twelve. Avulsion of the medial epicondyle may occur at any age up to the time of epiphyseal closure. Careful roentgenographic evaluation of these injuries is facilitated by having $\mathrm{x}$-ray films of both elbows.

Prior to epiphyseal closure, hyperextension of the elbow most often produces a supracondylar or dicondylar fracture of the distal part of the humerus with posterior displace-

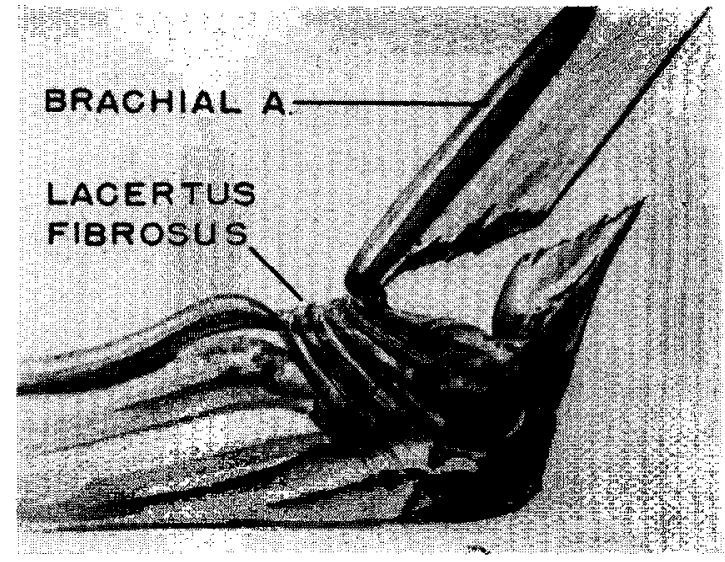

FIG. 9. Supracondylar fracture of the humerus with posterior displacement of the distal fragment.

ment of the distal fragment. Fractures of this type are usually associated with severe swelling and the threat of vascular embarrassment is always present. (Fig. 9.) The use of overhead skeletal traction with a Kirschner wire in the proximal end of the ulna and with the forearm suspended in a sling has enabled us to avoid the vascular complications of the supracondylar fracturc. Traction may bc utilized for a short period until swelling subsides and the arm can be placed in a splint, or the traction may be continued until callus appears. Three weeks is the usual period of traction. Manipulation of these fractures is seldom necessary in that the traction tends to reduce the fracture. Perfect functional results have consistently followed the use of this type of traction.

The epiphysis of the proximal end of the radius appears at about four years of age and unites with the shaft at fifteen years. Displacement of this epiphysis may occur up to the time of epiphyseal closure. Angular displacement can usually be reduced by closed manipulation, utilizing direct pressure on the displaced radial head. Severe displacement may require operative replacement. (Fig. 10.) The proximal radial epiphysis should not be excised.

\section{EPICONDYLITIS, TENDONITIS AND BURSITIS}

We have frequently encountered acute tendonitis and bursitis involving the radiohumeral bursa and the common tendon of origin of the forearm extensor muscles from the lateral humeral epicondyle (epicondylitis humeri). In 
Athletic Injuries to Elbow, Forearm, Wrist and $\mathrm{Hand}$

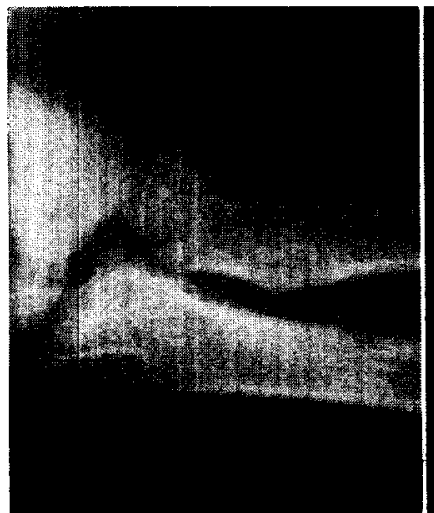

I OA

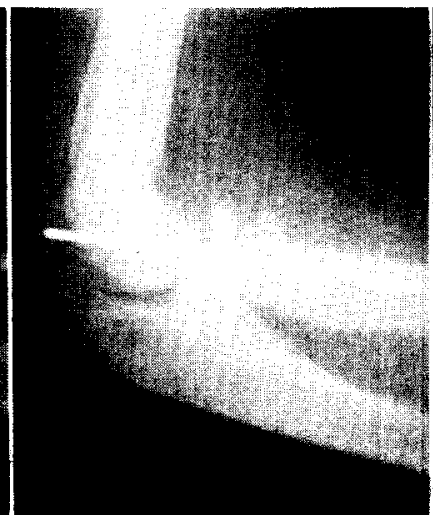

I OB

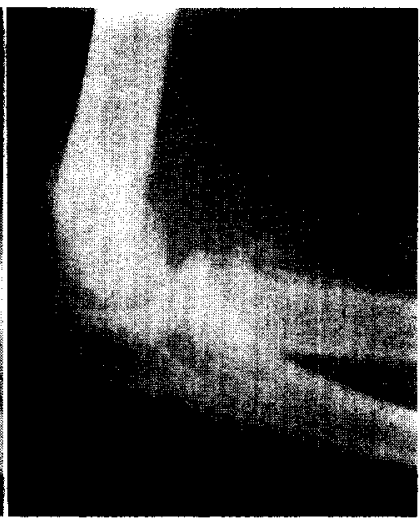

I $\mathrm{OC}$

Fic. Io. A, severe displacement of the proximal radial epiphysis in a thirteen year old boy. The fragment was very unstable. Closed reduction was unsatisfactory. B, open reduction was carried out. The unstable fragment was fixed with a Kirschner wire. C, after removal of the Kirschner wire. Callus is present around the radial neck.
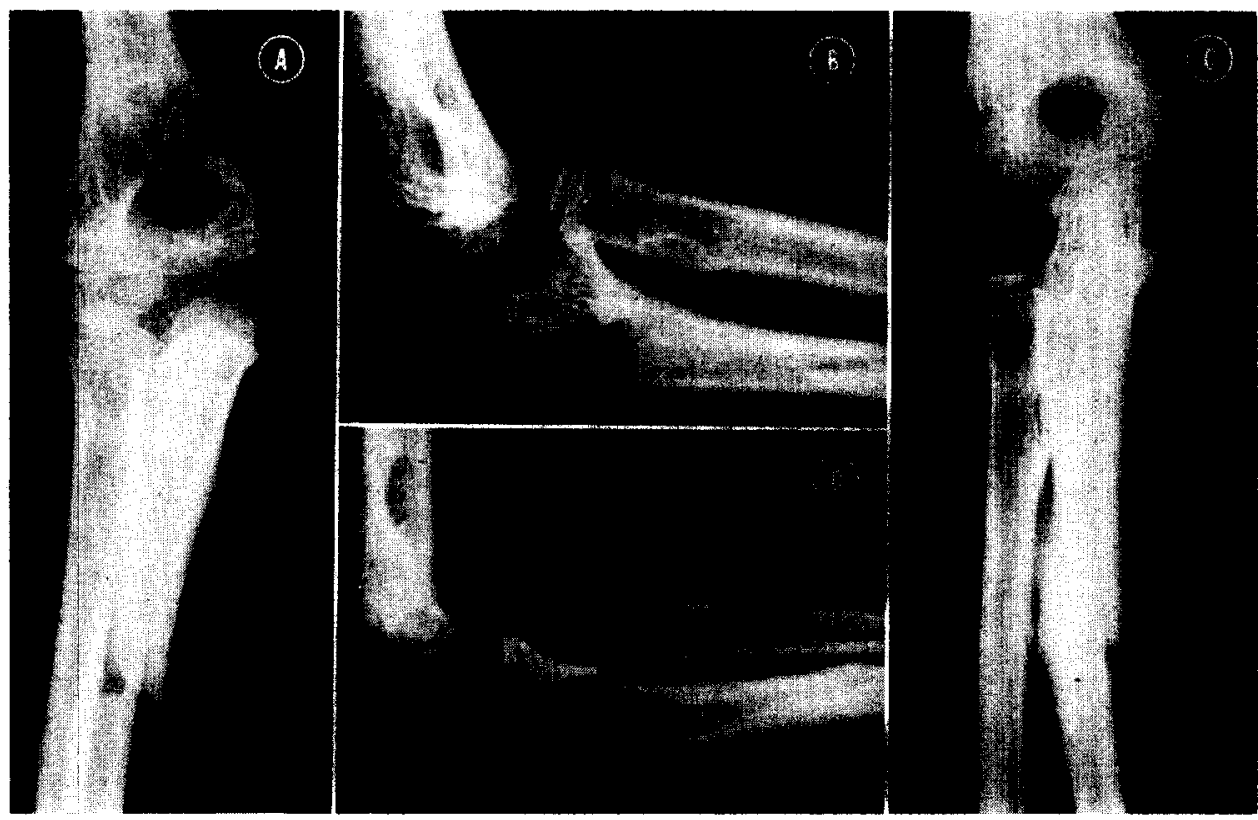

FIG. 11. A and B, anterior Monteggia injury (fracture of proximal part of the ulna and anterior dislocation of radial head) in an eight year old boy. $C$ and $D$, two months after reduction. The ulna is healed and the radial head is in normal alignment with the capitulum.

acute cases, severe point tenderness and swelling are noted directly over the epicondyle or over the radial head. Sports responsible for such irritations of the elbow are tennis, golf, fishing (fly casting), track (javelin throwing) and baseball (pitching). Repetitive movements of the elbow especially in pronation and supination seem to be a factor in producing epicondylitis humeri and radiohumeral bursitis. In addition to the localized pain and tenderness, radiating or referred pain may be felt in the forearm and hand. Pain may also be referred in a retrograde manner into the arm. Symptoms are increased by dorsiflexion of the wrist.

Conservative measures which have been useful have included rest with splinting and the application of moist heat to the elbow. The local injection of hydrocortisone has produced gratifying results in many cases.

Occasionally, deposits of calcium are seen in 


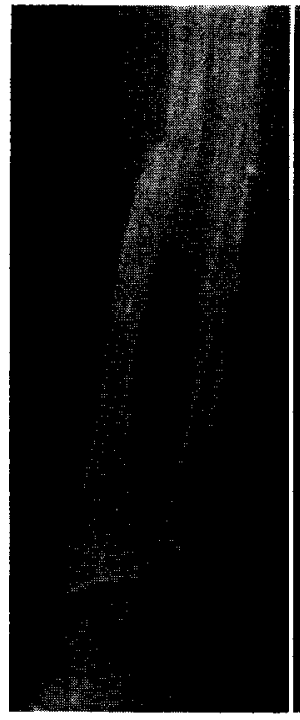

I $2 \mathrm{~A}$

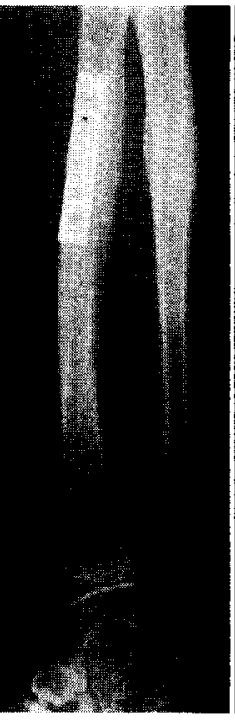

${ }_{12} \mathrm{~B}$

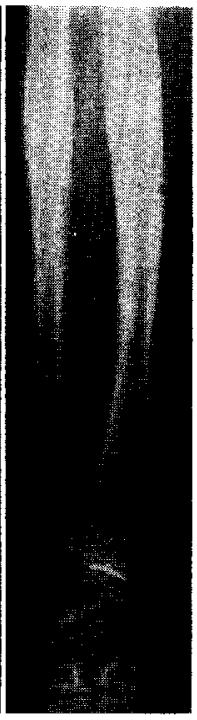

I $2 \mathrm{C}$
FIG. I2. A, complete transverse fracture of both radius and ulna in a fifteen year old boy. The fragments of the radius were rotated and locked so that a satisfactory reduction of the radius could not be obtained. B, open reduction and internal fixation of the radius were performed. $C$, nine months after injury both bones are healed and the plate has been removed.
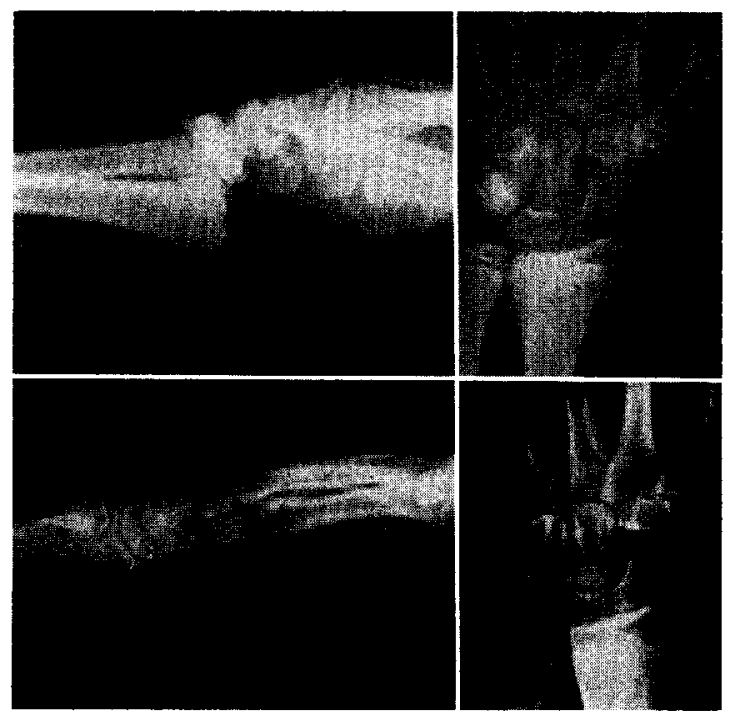

FIg. 13. Top, distal radial cpiphyscal separation in a fifteen year old boy. Bottom, three months after reduction. Closure of the epiphysis has taken place.

the soft tissue overlying the lateral humeral epicondyle. These are usually found in recurrent or chronic epicondylitis. Operative removal of the calcium has occasionally been necessary.
In resistant cases of epicondylitis, operative stripping of the common extensor tendon has provided relief.

\section{THE FOREARM}

Fractures of the bones of the forearm are relatively common in athletic injuries. Proximally, the ulna is often fractured along with anterior dislocation of the radial head in the so-called Monteggia injury. (Fig. I 1.) Hyperextension is the mechanism of this injury. Closed manipulative reduction followed by immobilization in plaster is the method of management. In the adult patient, closed reduction of the radial head may be incomplete because of interposition of the annular ligament. Open reduction with reconstruction of the annular ligament and intramedullary fixation of the ulna should be performed. Operative reduction should be carried out early. If operation is delayed, the danger of excessive ossification is present. The reverse or posterior Monteggia injury is very uncommon.

Direct blows to the ulnar aspect of the forearm will produce occasional isolated fractures of the ulna. These are usually undisplaced and respond wcll to immobilization in plaster. Greenstick fractures of both bones of the forearm are common in the junior high school and high school groups. Closed reduction and immobilization in plaster for eight to ten weeks will suffice.

Displaced fractures of both bones of the forearm prior to the age of epiphyseal closure should be treated with closed manipulation and cast. When a displaced fracture of the radius and ulna occurs in an athlete beyond the age of epiphyseal closure, open reduction and internal frxation with plate or intramedullary pin will give the best results. Even in the young, athletic patient, fractures of the shaft of the radius and ulna are slow to heal and the surgeon must be prepared to prolong immobilization in plaster until union is sound. (Fig. I 2.) Delayed union of both bones of the forearm should be treated by early bone grafting.

Injuries to the distal end of the forearm may produce damage to the distal radial epiphysis. Such injuries are uncommon after twelve years of age. Hyperextension commonly causes dorsal displacement of the distal radial epiphysis. Frequently, a small triangular fragment of bone from the dorsal radial cortex remains attached to the epiphysis. (Figs. I3 and I4.) Closed 
Athletic Injuries to Elbow, Forearm, Wrist and Hand

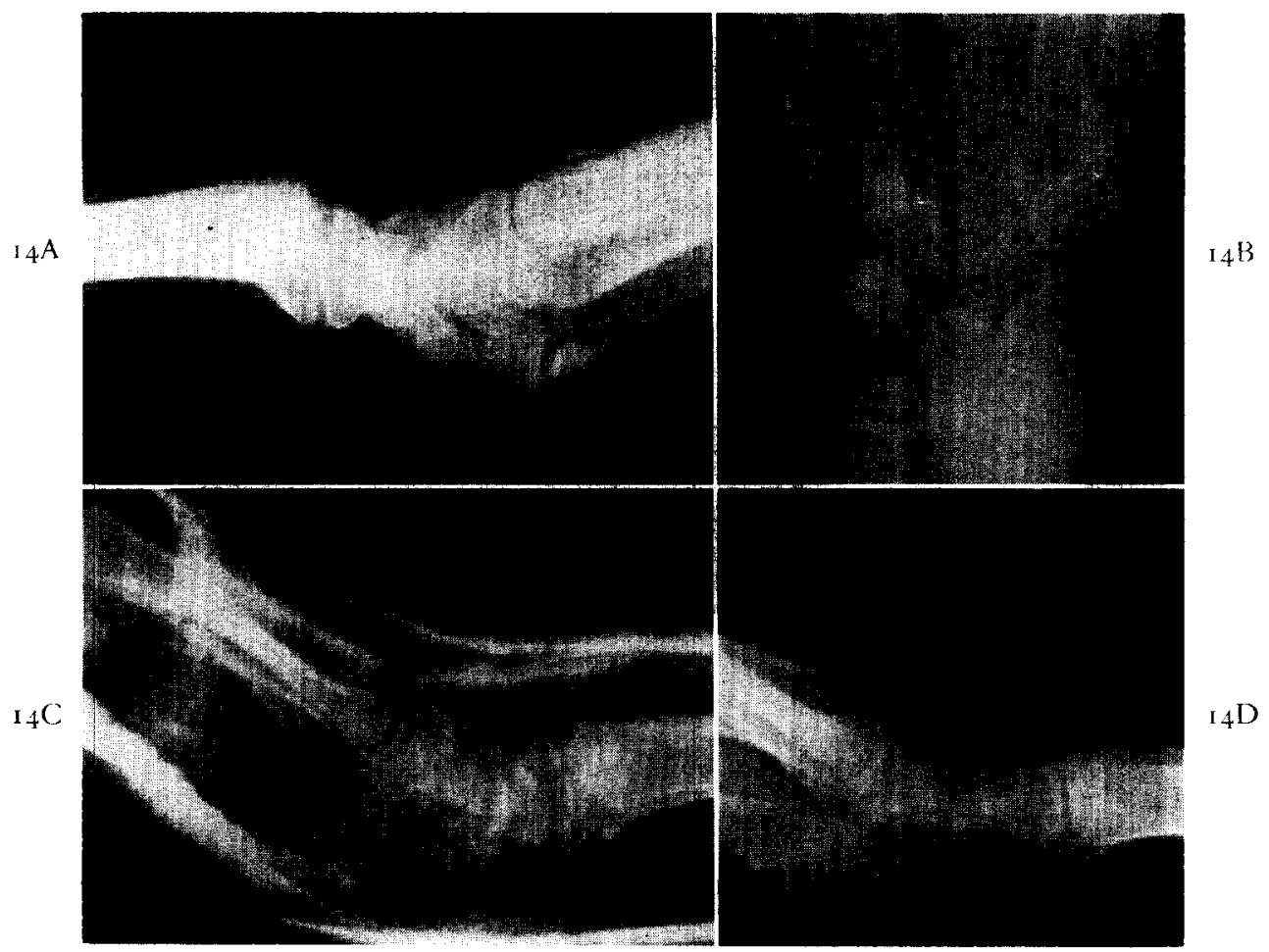

Fic. I 4. A and B, volar displacement of the distal radial epiphysis. C, following reduction, the wrist is held dorsiflexed in plaster. D, six weeks after injury the radius is healed.

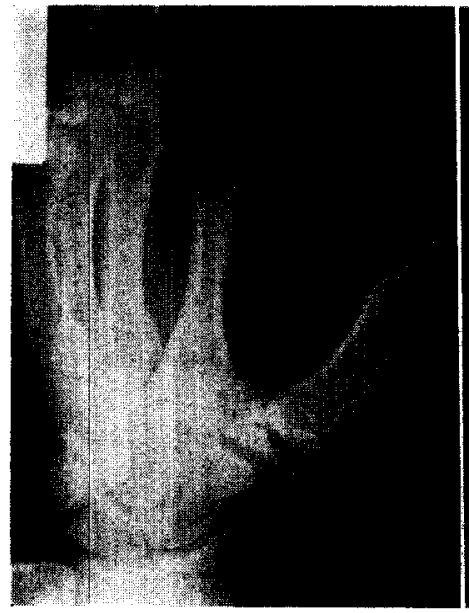

$1 ; \mathrm{A}$

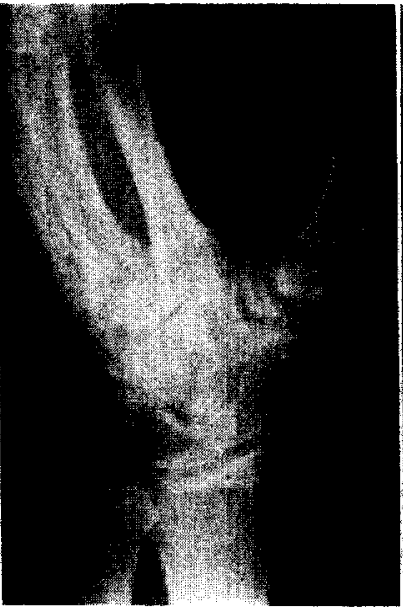

${ }_{15} \mathrm{~B}$

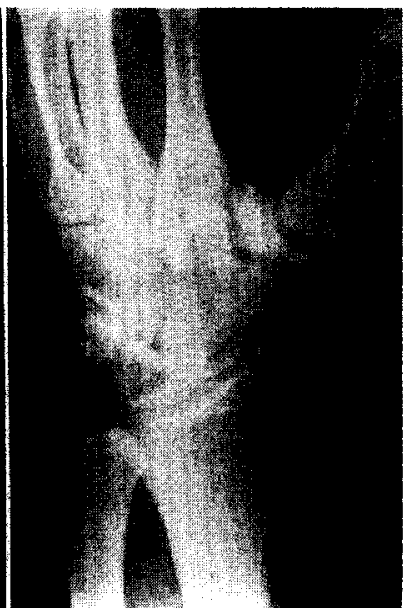

${ }_{15} \mathrm{C}$

FIG. I 5. A, a university football player sustained a blow to the outstretehed thumb. There is a comminuted fracture of the greater multangular bone. B, two months after injury. The greater multangular bone is healing. $C$, one year after injury. Early traumatic arthritis is present in the carpometacarpal joint.

reduction is not difficult unless complete displacement of the epiphysis with overriding is present. A disturbance of growth following distal radial epiphyseal separation is rare unless there has been direct damage to the epiphysis causing a fracture through the epiphysis or crushing of the epiphyseal plate. (Fig. 13.) Special care on the part of the surgeon to obtain reduction of the displaced epiphysis by a single manipulation is essential. Repeated 


\section{Badgley and Hayes}

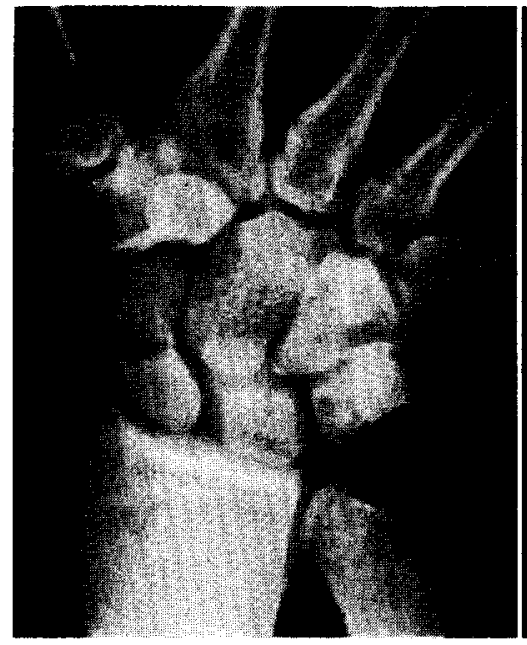

$16 \mathrm{~A}$

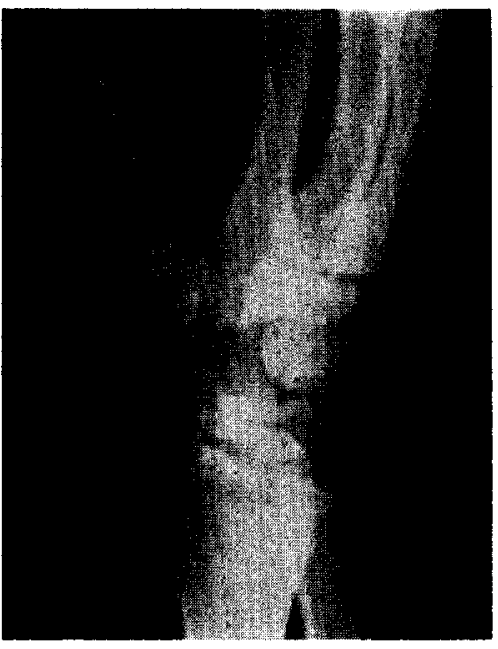

$16 \mathrm{~B}$

FIG. I6. A, fracture through the waist of the carpal scaphoid bone. B, frve months after injury, the fracture is healed.

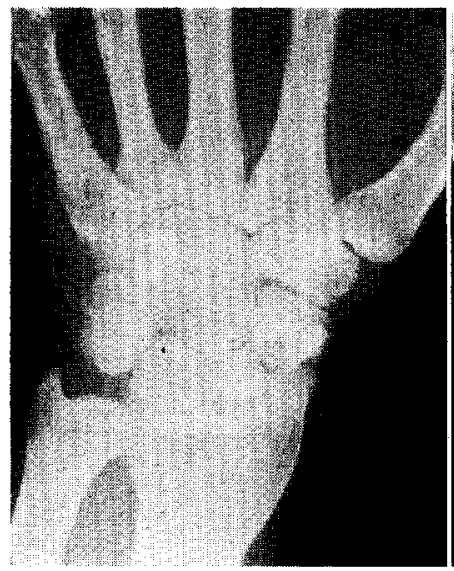

$17 \mathrm{~A}$

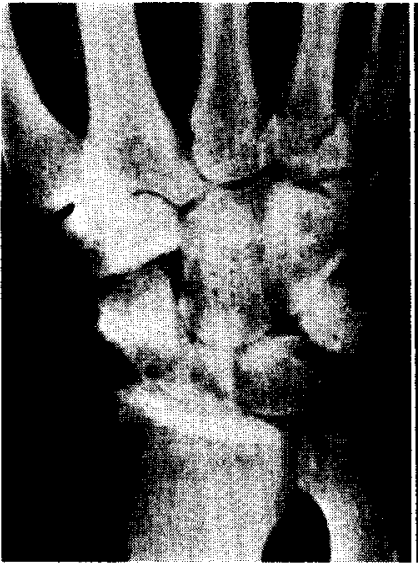

$17 \mathrm{~B}$

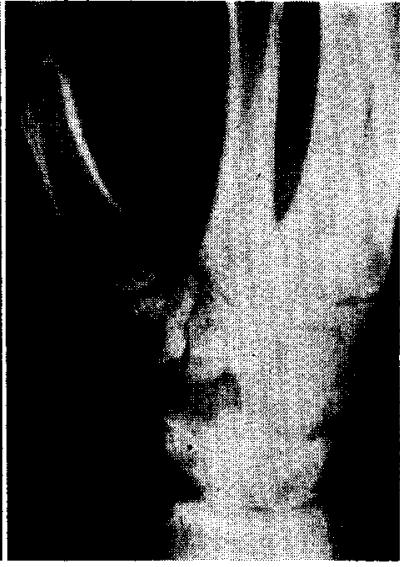

$17 \mathrm{C}$

Fig. I7. A, non-union of carpal scaphoid. Arthritic changes are present between the scaphoid and the radial styloid. $\mathrm{B}$ and $\mathrm{C}$, after excision of the styloid process of the radius and autogenous graft of the scaphoid. The graft was taken from the distal radius.

manipulations are apt to cause damage to the epiphyseal plate and subsequent abnormality in growth.

\section{CARPAL INJURIES}

The surgeon attending athletes must be prepared to manage a great variety of carpal injuries. Although fracture of the carpal scaphoid bone is the most common carpal fracture, we have seen isolated fractures of each of the carpal bones. Failure to recognize such fractures and to immobilize the wrist may lead to nonunion and traumatic arthritis. (Fig. Is.)
It must be emphasized that a fracture line may not be visible immediately after injury in any of the usual routine four to six $x$-ray views of the wrist. These injuries should not be dismissed as "sprains," but should be immobilized as fractures of the carpal scaphoid for two weeks. Another set of roentgenograms then, with the carpus out of plaster, will very often reveal a fracture line. Sprain of the wrist is therefore a diagnosis of exclusion.

In young athletic males, carpal scaphoid fractures will usually heal if immobilized for an adequate period. (Fig. I6.) Non-union is usually the result of an unrecognized fracture or an 
Athletic Injuries to Elbow, Forearm, Wrist and Hand

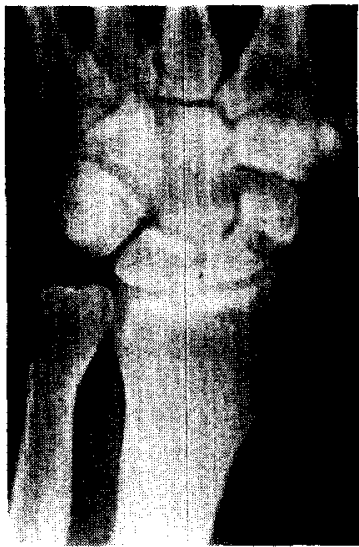

$18 \mathrm{~A}$

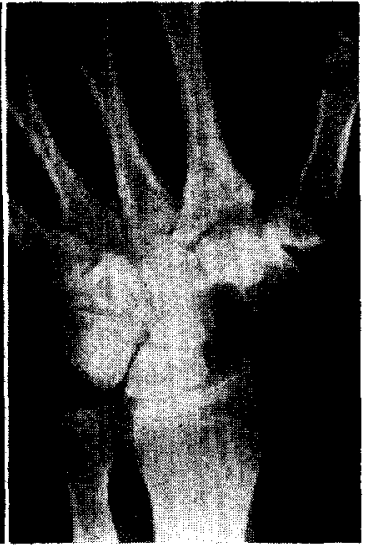

I $8 \mathrm{~B}$

Fir. I8. A, non-union of carpal scaphoid twenty-one months after injury. There is localized intercarpal arthritis. B, ten months after excision of the carpal scaphoid.

inadequate period of immobilization. Ununited carpal scaphoid fractures commonly exhibit associated traumatic arthritis involving the radiocarpal joint or the adjacent intercarpal joints. When non-union is present in a carpal scaphoid fracture, two basic operative approaches are available to the surgeon. Excision of the styloid process of the radius along with autogenous grafting of the navicular has been used. (Fig. 17.) The graft may be taken from the distal radius, the tibia or the ilium. Excision of the carpal scaphoid has given consistently good results in this clinic. (Fig. I8.) Excellent functional results in outstanding athletes have been obtained. In cases of severe traumatic arthritis of the carpus, fusion of the wrist must be considered.

Carpal dislocations with or without fracture will occasionally be seen in athletic injuries of the wrist. Severe hyperextension may produce anterior dislocation of the carpal lunate. (Fig. 19.) Recluction by closed technic should be attempted, but, if not successful, should be followed by prompt open reduction. Repeated manipulative attempts at closed reduction of the anteriorly dislocated lunate may lead to damage to the median nerve. An unreduced dislocation of the carpal lunate may lead to interference with the median nerve and the causalgia syndrome. Following reduction of the lunate, a carpal tunnel syndrome may develop secondary to swelling.

Severe hyperextension and radial deviation of the carpus causes the navicular to impinge

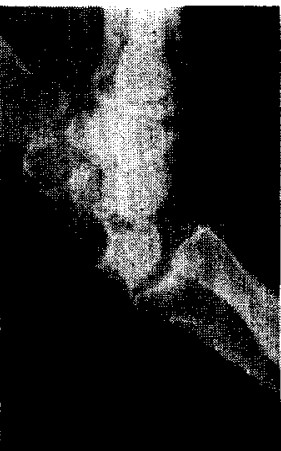

$19 \mathrm{~A}$

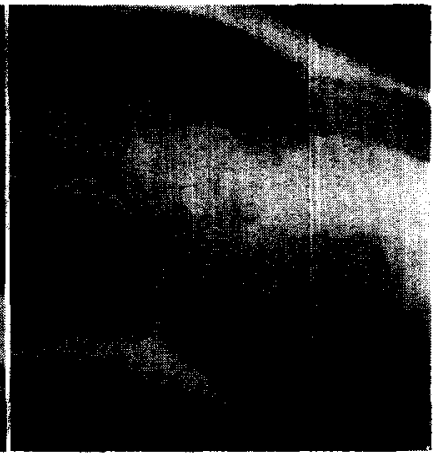

$19 \mathrm{~B}$
FIg. I9. A, anterior dislocation of the carpal lunate bone. $\mathrm{B}$, after reduction in plaster, the lunate is restored to its normal position in the wrist.

upon the radial sty loid. Trans-scaphoid perilunar fracture dislocation of the carpus often follow's this injury. The scaphoid is fractured through its waist. The proximal scaphoid fragment and the lunate remain in the normal relationship with the radius while the distal scaphoid fragment and the remainder of the carpal bones are displaced as a unit in a dorsoradial direction. Occasionally, the radial styloid is also fractured in this injury. (Fig. 20.)

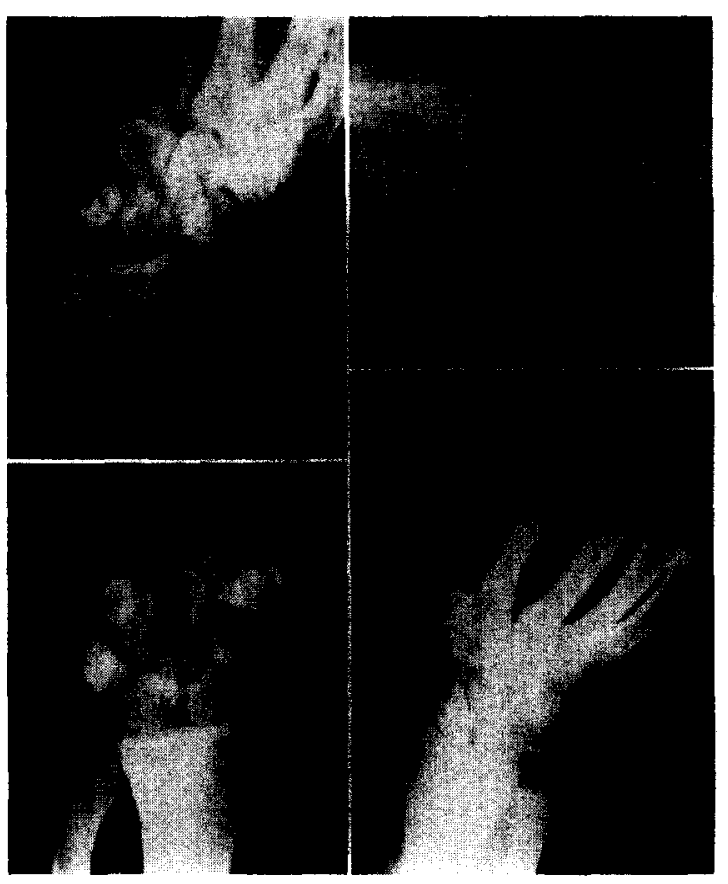

Fig, 20. Top, trans-scaphoid perilunar fracture dislocation of the wrist. The radial and ulnar styloid processes are also fractured. Bottom left, immediately after reduction. Bottom rigbt, six months after injury the fractures are healed. 


\section{Badgley and Hayes}

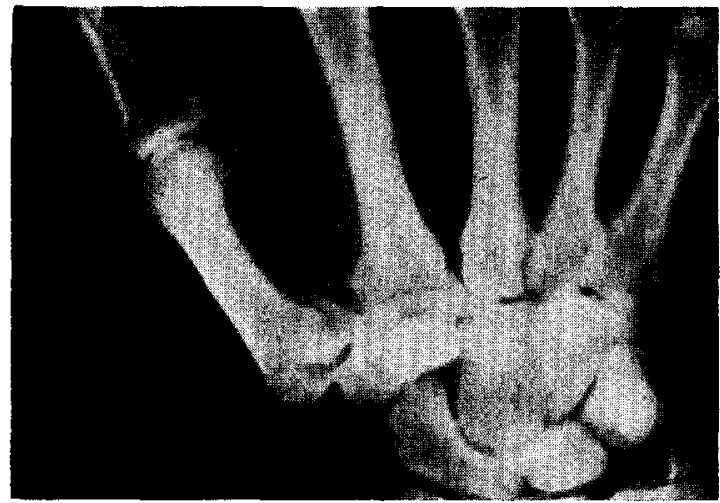

sents less than one-third of the navicular, ard aseptic necrosis is present, it should be excised.

\section{INJURIES TO THE HAND}

Metacarpal Injuries. Bennett's fracture of the hase of the frrst metacarpal is quite common in athletic injuries. A blow on the tip of the outstretched thumb tends to force the first metacarpal proximally. The unique shape of the base of the first metacarpal with its saddle-like configuration makes it prone to fracture. The fracture separates the projecting medial lip of the metacarpal base and allows proximal subluxation of the first metacarpal on the greater multangular bone. Reduction of this fracture dislocation can be accomplished by traction and abduction of the thumb. (Fig. 21.) Skeletal traction utilizing a Kirschner wire through the first phalanx is often necessary to maintain a good reduction. After four weeks, traction may be discontinued. Occasionally operative reduction is advisable. Fixation with screw or Kirschner wire may be used.

Metacarpal fractures can be grouped according to the configuration of the fracture. Transverse fractures of the shaft and fractures at the base of metacarpals two through five are reduced and immobilized for four weeks. Immobilization is accomplished with a short arm cast incorporating a curved, metallic, padded splint under the involved finger. (Fig. 22.) The finger is placed in a flexed position of function. If a metacarpal fracture is oblique and tends to override or angulate, skeletal traction is utilized with a Kirschner wire through the middle or distal phalanx. After three weeks, traction may be discontinued and the hand immobilized in a cast with the involved finger supported on a metallic splint in a flexed position.

Fractures through the metacarpal neck with

Fig. 2I. Top. Bennett's fracture of base of first metacarpal. Middle, reduction maintained in plaster cast. Bottom, four weeks after injury, the fracture is healed.

Closed reduction and immobilization in a short arm plaster including the thumb should be carried out until union of the scaphoid fracture is sound. Aseptic necrosis of the lunate following dislocation may occur. Excision of the necrotic bone is indicated. If aseptic necrosis of the proximal fragment of a fractured scaphoid occurs, bone grafting will assist revascularization. If the proximal fragment repredepression of the head of the metacarpal are often troublesome to reduce. Acute flexion of the finger with the metacarpophalangeal joint flexed 90 degrees allows the surgeon to control the head of the metacarpal, Upward pressure on the metacarpal head through the proximal phalanx will lift the dropped metacarpal head. (Fig. 23.) Caution must be used to avoid necrosis of the skin over the proximal interphalangeal joint, however.

Digital Injuries. Phalangeal fractures are managed by immobilizing the digit in flexion 


\section{Athletic Injuries to Elbow, Forearm, Wrist and Hand}

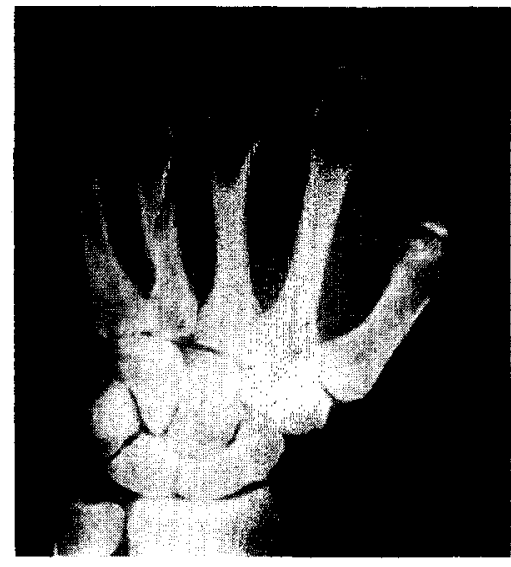

$22 \mathrm{~A}$

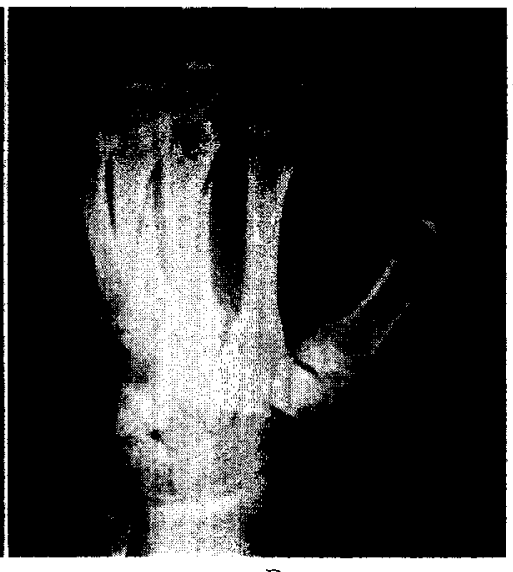

$22 \mathrm{~B}$

Fig. 22. $A$ and $B$, oblique fractures of the third and fourth metacarpals with. minimal overriding.

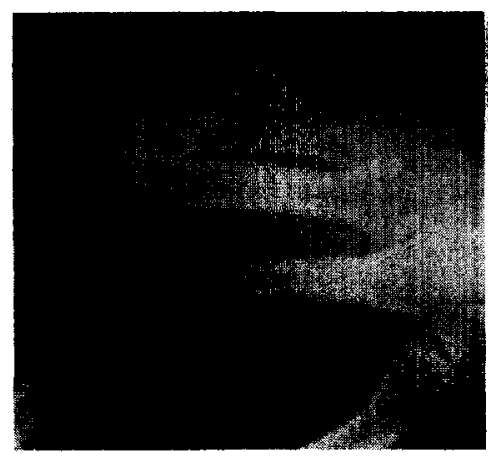

$23 \mathrm{~A}$

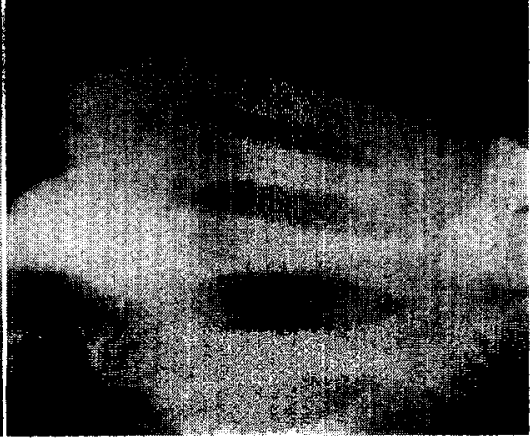

${ }_{23} \mathrm{~B}$

FiG. 23. A, fracture of the neck of the fifth metacarpal with depression of the head. B, after reduction and application of a splint. The dropped head has been corrected. Slight Iateral displacement persists.

on a padded, malleable metal splint for two to three weeks.*

Dislocations of the metacarpophalangeal joints and interphalangeal joints are extremely common in athletic injuries. In simple posterior dislocations, the collateral liganients are relatively intact, and after reduction splinting in flexion for seven to ten days will suffice. However, if lateral displacement of the joint has taken place, one of the collateral ligaments must have been disrupted and careful treatment must be carried out. (Fig. 24.)

* Reduction of fractures of proximal phalanges and unsupported metacarpals can be maintained and in addition a fair range of active motion obtained by the use of a simple skeletal traction hook inserted through the dorsal cortex of the middle phalanx. (Quigley, T. B. and URist, M. R. Interphalangeal joints. Am. J. Surg., 73 : $175,1947$. )
Accurate reduction and retention of reduction for at least three weeks are indicated. If any residual lateral instability is noted, open repair of the collateral ligament is in order. Neglected lateral instability will lead to a disabling, unstable joint. (Fig. 25.)

Injuries of the hand, other than fractures or dislocations, are very common. We have encountered isolated ruptures of flexor tendons in baseball, basketball and football players. Prompt repair utilizing the Bunnell pull-out wire technic is ideally suited for repair of these tendons. Ruptured long extensor tendons may be repaired with the Bunnell frgure eight steel wire technic.

The so-called "baseball finger" represents one of the most common digital injuries. Avulsion of the extensor mechanism from the 


\section{Badgley and Hayes}

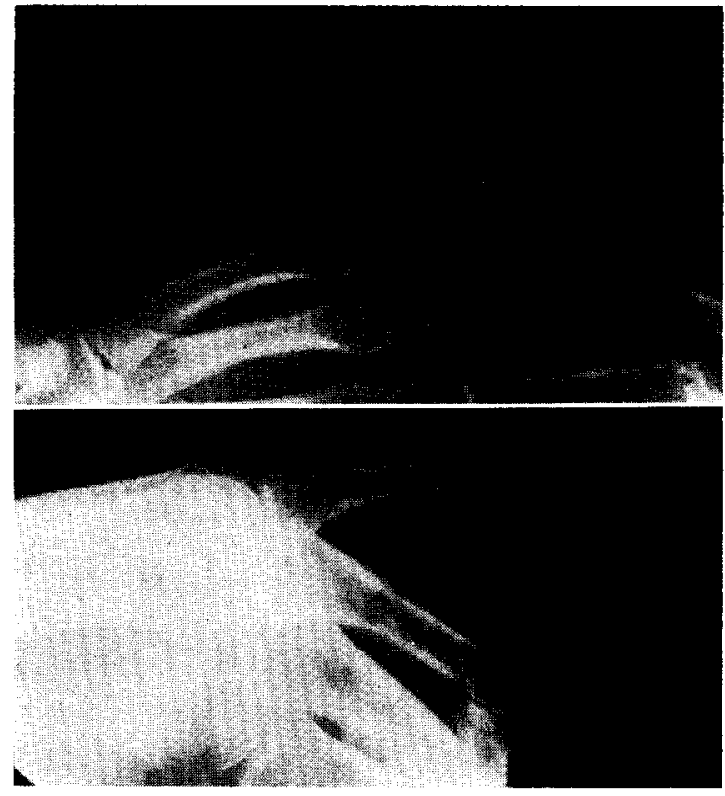

FIG. 24. Top, compound dislocation of the proximal interphalangeal joint of the little finger. Botlom, three weeks post injury. The collateral ligaments are healed.

distal phalanx causes a flexion deformity of the distal phalanx. Frequently, a triangular fragment of bone is avulsed from the dorsal tip of the base of the distal phalanx. Although the closed splinting technic with the proximal interphalangeal joint in flexion and the distal interphalangeal joint in extension has given good results in many instances, operative repair has produced uniformly good results and avoids the hazard of necrosis of the skin. If a small fragment of bone has been avulsed from the distal phalanx, the splinting technic will succeed if satisfactory reduction of the avulsed fragment can be obtained. In the absence of an avulsion fracture, opposition of the torn tendon fragments is uncertain and open repair will ensure a good result. If the closed technic is elected, splinting is carried out for six weeks. Lateral $x$-ray films will confirm retention of reduction of the avulsed fragment. Operative repair of the tendon allows the time of splinting to be shortened to three weeks.

Rupture of the extensor tendon to a finger at its insertion into the base of the middle phalanx allows the so-called "boutonniere" deformity with flexion of the proximal interphalangeal joint and extension of the metacarpal phalangeal and distal interphalangeal joints. Repair by the figure eight stainless steel wire technic is followed by splinting for three to four weeks.

\section{COMPLICATIONS}

The surgeon treating athletic injuries must be constantly aware of the major complications which may result from such injuries. Open wounds must be thoroughly débrided and irrigated prior to closure. The value of delayed

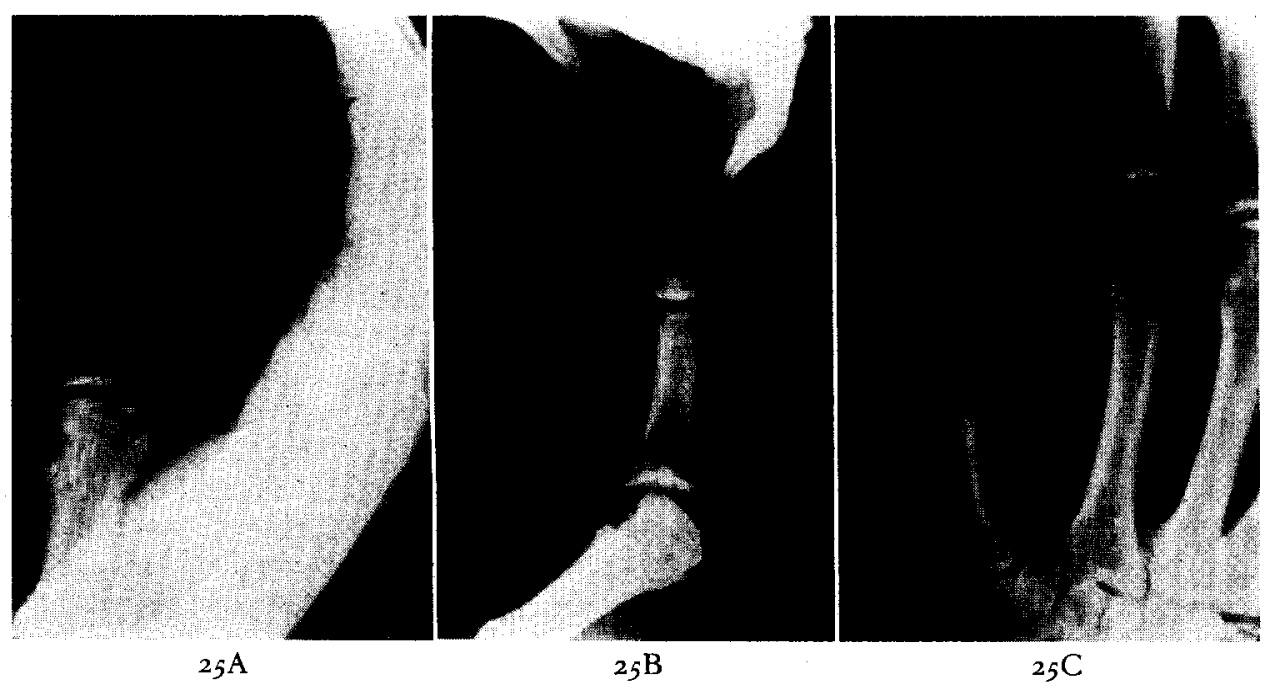

FIG. 25. A and B, old neglected dislocation of the metacarpophalangeal joint of the thumb. Marked lateral instability is present which interferes with pinch and grasp functions. C, after reconstruction of the collateral ligaments, the thumb was stable for a short period. Recurrence of instability made arthrodesis of this joint necessary. 


\section{Athletic Injuries to Elbow, Forearm, Wrist and Hand}

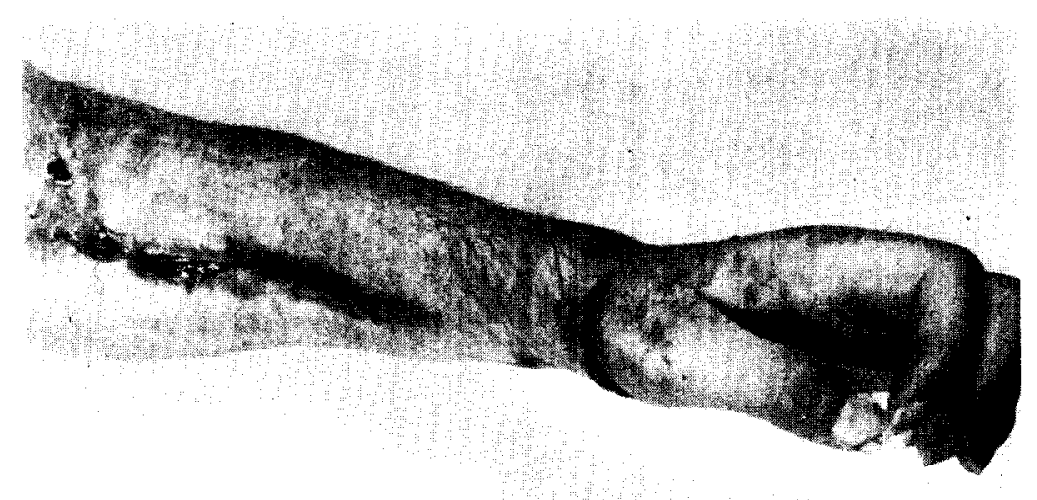

Fig. 26. Severe Volkmann's ischemia of forearm and hand following fracture of the forearm bones sustained in football. Damage was so severe, amputation was necessary.

primary closure in badly contaminated wounds or in wounds with extensive contusion of tissue should not be overlooked. Vascular and neural complications of fractures and dislocations must be constantly kept in mind. We have seen an extremely severe Volkmann's ischemia of the forearm following a closed fracture of radius and ulna sustained in a high school football game. (Fig. 26.) Compression neuropathy of the median nerve has been seen in injuries of both the elbow and wrist. Delayed ulnar neuritis has been seen in an old valgus deformity of the elbow. (Fig. 27.)

Injuries to the epiphyses have the potentiality of giving rise to future disturbances of growth. In general, epiphyseal separations in which the epiphyseal plate is neither fractured nor crushed are not followed by the arrest of growth. However, fractures which traverse the epiphyseal plate or which cause a crushing injury to the epiphyseal plate will often produce the arrest of growth. Young athletes with epiphyseal injuries must be followed up closely with repeated careful measurements and $x$-ray examination to determine whether or not growth has been disturbed. This is of particular importance in injuries of the wrist and elbow. Premature closure of the distal radial epiphysis will lead to an unpleasant deformity of the wrist with radial deviation of the hand and prominence of the distal ulna. Premature asymmetrical closure of the distal humeral epiphysis leads to a valgus or varus deformity of the elbow depending on the lateral or medial location of the humeral injury.

Traumatic arthritis may follow any intra- articular injury. Osteochondritis dissecans of the elbow usually involves the capitulum of the humerus. Traumatic arthritis is very prone to develop in the wrist in the presence of an ununited fracture of the carpal scaphoid.

In all athletic injuries, early active exercises will minimize loss of joint motion. Active exercises for muscle strengthening and range of motion should be started at the earliest possible moment. Even after a severe dislocation of the elbow, active exercise is begun after three weeks' immobilization. Passive stretching is never allowed. In the presence of myositis

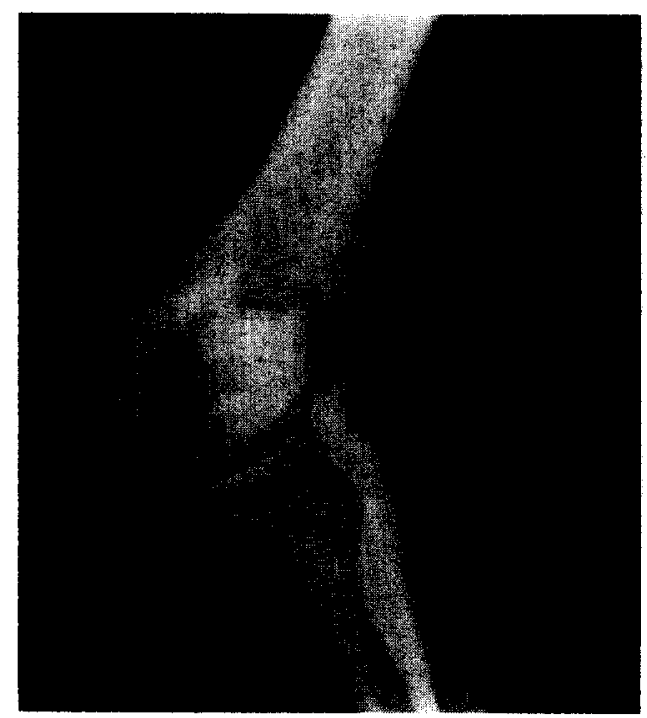

Fig. 27. Cubitus valgus secondary to an old ununited fracture of the Iateral condyle of the humerus. Neuritis of the ulnar nerve was treated by anterior transplantation of the nerve. 


\section{Badgley and Hayes}

ossificans, active exercise is encouraged and the bony mass grows smaller and matures. (Fig. 6.)

Athletic injuries may be neglected for one of several reasons. Occasionally a player will conceal an injury in order to avoid being taken from the lineup. The attending surgeon should emphasize to coaches, trainers and players the necessity of a careful and thorough evaluation of all injuries. Only by prompt and effective treatment can the surgeon restore the injured part to a good functional status.

\section{REFERENCE}

I. Murray, C. R. The timing of the fracture healing process. J. Bone of Joint Surg., 23: 598-606, 1941. 